\title{
Effect of potential vorticity on flow rate through a gap
}

\author{
J. A. Whitehead \\ Department of Physical Oceanography, Woods Hole Oceanographic Institution, Woods Hole, Massachusetts, USA
}

Received 17 September 2004; revised 24 March 2005; accepted 14 April 2005; published 16 July 2005.

[1] Mathematical solutions for constant potential vorticity critically controlled flow through ocean passages are complicated and not available in simple form. Therefore, to provide formulas for numerical circulation and ocean climate models, two simple formulas for volume flux are developed here. They are fitted to numerical values of the critical flux for constant potential vorticity flow over a flat bottom through a constriction. The two formulas of increasing complexity agree with the numerical values to better than $6 \%$ and $1.4 \%$. These flux values are up to $24 \%$ less than the values of flux from zero potential vorticity formulas presently applied to ocean passages. The most precise new formula is used to predict flux magnitude through nine ocean passages that have current meter measurements. The size of the revisions compared to zero potential vorticity predictions is a few percent in the direction of better agreement. For further improvement between prediction and observation, other factors such as realistic bottom topography, friction, mixing, waves, and eddies must be included.

Citation: Whitehead, J. A. (2005), Effect of potential vorticity on flow rate through a gap, J. Geophys. Res., 110, C07007, doi:10.1029/2004JC002720.

\section{Introduction}

[2] The very deepest portions of the main bodies of the oceans (exclusive of trenches) consist of a number of large basins greater than $5000 \mathrm{~m}$ deep separated by topography with the deepest sill at some shallower depth. The very coldest and densest ocean water in the world migrates from deep basin to deep basin by filling the basin closest to the cold-water source and then overflowing through the deepest passage into the next basin. This basin is filled in a similar manner, and the cold water leaves through the deepest exit passage of that basin as an overflow. This sequence continues for a number of basins.

[3] Since the volume flux of a layer of water exiting a basin through a passage is an important variable to know in physical oceanography, for over 30 years simple formulas based on "rotating hydraulics" dynamics have been devised and used to estimate flux through gaps between large ocean basins. The typical model is a large basin filled with dense water with a passage to another basin. The dense water exits by gravity flow. Most extant theories assume inviscid dynamics of a rotating fluid. The solutions are generally quite complicated to find analytically. One case in which the basin fluid is infinitely deep compared to the depth of fluid in the passage [Whitehead et al., 1974] is referred to as the zero potential vorticity solution. Those formulas for volume flux are quite simple and easy to use for predicting flow through deep ocean passages [Whitehead, 1989, 1995, 1998]. Although they have been applied to various passages in the ocean, the case in which the bottom of the layer of dense fluid in the upstream basin has finite depth, rather

Copyright 2005 by the American Geophysical Union. 0148-0227/05/2004JC002720\$09.00 than infinite depth, is clearly more realistic since ocean water is stratified. That case is referred to as the constant potential vorticity case. Here we show that the inclusion of constant potential vorticity makes small changes (generally $<20 \%$ ) to the zero potential vorticity volume flux estimates. In addition, corrections applied to ocean examples are even smaller than $20 \%$ because most ocean passages have a width different from the widths with the greatest changes.

[4] Unfortunately, the general problem of flux as a function of passage width for critically controlled flow over a flat bottom is complicated enough to make analytic formulas difficult to simplify. Most cases have only been numerically calculated [Gill, 1977; Pratt and Armi, 1987; Whitehead and Salzig, 2001]. Such a computation involves resolving the flow to the equivalent of a kilometer or less in the lateral direction for a typical ocean, and this is a finer scale than is easily achieved either in most oceanographic field programs or by numerical models of general ocean circulation. What would be more useful for field oceanographers or numerical modelers is a simple formula to estimate flux better than the zero potential vorticity flow. These are developed here for constant potential vorticity flow.

[5] Nine ocean locations have current meter data extensive enough to measure a long time averaged flux. These locations are listed in Table 1. The magnitudes of volume flux with our new formulas at each location will be compared here with the zero potential vorticity formula and with ocean measurements.

\section{Method}

[6] The objective is to produce two simple empirical formulas that agree with the numerical calculations of the 
Table 1. Data and Fluxes of Nine Ocean Passages

\begin{tabular}{|c|c|c|c|c|c|c|c|c|c|}
\hline Name & $\Delta \rho^{*} / \rho^{*} \times 10^{4}$ & $D^{*}, \mathrm{~m}$ & $f^{*} \times 10^{4}, \mathrm{~s}^{-1}$ & $w^{*}, \mathrm{~km}$ & $W$ & $Q^{*}(0), \mathrm{Sv}$ & $Q_{2}^{*}, \mathrm{~Sv}$ & $Q_{o b s}^{*}, \mathrm{~Sv}$ & $\begin{array}{c}\text { Flux } \\
\text { Measurements } \\
\text { Source } \\
\end{array}$ \\
\hline Anagada Passage & 0.45 & 165 & 0.45 & 10 & 1.65 & 0.14 & 0.12 & 0.11 & MacCready et al. [1999] \\
\hline Discovery Gap & 0.1 & 600 & 0.87 & 80 & 28.4 & 0.2 & 0.21 & 0.21 & Saunders [1987] \\
\hline Romanche Fracture Zone & 0.73 & 380 & 0.02 & 20 & 0.08 & 2.15 & 2.09 & 0.66 & Mercier and Speer [1998] \\
\hline Faroe Bank Channel & 5.0 & 400 & 1.3 & 20 & 1.84 & 2.62 & 2.81 & 1.9 & Saunders $[1990]$ \\
\hline Ceara Abyssal Plain & 0.5 & 430 & 0.1 & 700 & 15.0 & 4.53 & 4.62 & 2 & Hall et al. [1997] \\
\hline Vema Gap & 0.5 & 1000 & 0.28 & 9 & 0.36 & 3.35 & 3.03 & 2.1 & McCartney et al. [1991] \\
\hline Denmark Strait & 3.0 & 580 & 1.3 & 350 & 34.5 & 3.8 & 3.88 & 2.9 & Dickson et al. [1990] \\
\hline Vema Channel (with GEOSECS data) & 1.0 & 1540 & 0.7 & 446 & 25.2 & 16.6 & & 4 & Hogg et al. [1999] \\
\hline Vema (better data) & 1.0 & 1100 & 0.7 & 446 & 29.8 & 8.62 & 8.64 & 4 & Hogg et al. [1999] \\
\hline Samoa passage & 0.3 & 1050 & 0.23 & 240 & 9.8 & 7.05 & 7.19 & 6 & Rudnick [1997] \\
\hline
\end{tabular}

constant potential vorticity solutions to successively greater precision. The governing equations are well known [Gill, 1997] and will not be reviewed here except to say that they are steady semigeostrophic equations for a layer of rotating fluid beneath a less dense motionless fluid. Upstream, there is a wide basin with a flat bottom and stagnant fluid layer, with boundary currents along one or both walls. This is the standard case for constant potential vorticity. The fluid flows through a passage of finite width with a flat bottom at a different depth and then into a deeper downstream basin. Flow is critically controlled in the passage.

[7] There are a number of parameters describing this problem. The presence of rotation dictates two depths. These will be considered fixed in the upstream basin. The first depth is $D_{r}^{*}=\left(v_{u r}^{*^{2}} / 2 g^{*^{\prime}}\right)+d_{u r}^{*}$, (dimensional quantities are starred, nondimensional ones are not). This is elevation of the Bernoulli "head" on the right-hand wall above the bottom of the upstream basin, in which $v_{u r}^{*}$ is velocity along that wall, $d_{u r}^{*}$ is elevation of the water surface at the upstream right-hand wall, and the acceleration of the surface of the fluid layer from gravity (reduced gravity) is $g^{*^{\prime}}=$ $g^{*} \Delta \rho^{*} / \rho^{*}$, where the layer has density $\rho^{*}+\Delta \rho^{*}$ and a fluid extending up to infinity above the layer has density $\rho^{*}$. The second length scale is $D_{\infty}^{*}$, which is depth of stagnant fluid in the upstream basin. A region with such stagnant fluid exists in the interior if the upstream basin is very wide since in that case, boundary layers along the sidewalls transport all of the constant potential vorticity fluid. The change in depth from the upstream basin floor to the passage floor $\Delta h^{*}$ gives a third geometric length. Finally, the passage has width $w^{*}$. The other two parameters of relevance are reduced gravity $g^{*^{\prime}}$ and the Coriolis parameter from earth rotation $f^{*}$.

[8] Following notation used by Whitehead [2003], we select a depth scale $D^{*}=D_{r}^{*}-\Delta h^{*}$. This is divided by depth of stagnant upstream fluid to give the first important dimensionless number called the dimensionless potential vorticity $q=\left(D_{r}^{*}-\Delta h^{*}\right) / D_{\infty}^{*}$. A second dimensionless number is width of the passage divided by Rossby radius $W=w^{*} f^{*} / \sqrt{g^{* \prime} D^{*}}$, and the third dimensionless number is $d_{r}=d^{*}{ }_{r} / D^{*}$, where $d_{r}^{*}$ is depth of the fluid above the floor of the passage along the right-hand wall of the passage. Since there are two dimensions, length and time, using the parameters $D^{*}, D_{\infty}^{*}, d_{r}^{*}, w^{*}, g^{*}, f^{*}$, and volume flux $Q^{*}$, the Buckingham pi theorem gives that the number of dimensionless numbers is $7-2$, or 5 . This reduces to 4 because the semigeostrophic approximation requires that the so-called hydrostatic number is small, $f^{*} D^{*} / \sqrt{g^{\prime *} D^{*}} \ll 1$.
The four dimensionless parameters $q, W, d_{r}$, and a dimensionless flux $Q=f^{*} Q^{*} / g^{* \prime} D^{* 2}$ are all related together in equation (2.12) of Whitehead and Salzig [2001], which in this notation is

$$
\begin{aligned}
Q\left(d_{r}, q, W\right)= & \frac{1}{2} d_{r}^{2}-\frac{1}{2}\left[q^{-1}-\frac{1}{2}\left(q^{-1}-d_{r}+\sqrt{2 q^{-1}\left(1-d_{r}\right)}\right)\right. \\
& \left.\cdot e^{\sqrt{q} W}-\frac{1}{2}\left(q^{-1}-d_{r}-\sqrt{2 q^{-1}\left(1-d_{r}\right)}\right) e^{-\sqrt{q} W}\right]^{2}
\end{aligned}
$$

[9] The critically controlled flux through a passage is found by determining the value of $d_{r}$ for $\frac{\partial Q}{\partial d_{r}}=0$, and using it to calculate a value for maximum flux. The zero potential vorticity critically controlled flux [Whitehead et al., 1974] is found in the limit of $q \rightarrow 0$ and it has a simple solution

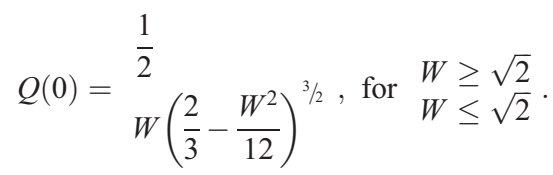

Values of $Q(0)$ for $0 \leq W \leq 4$ are shown in Figure 1 .

[10] A simple formula for flux for $q=1$ is not known. As a substitute, we fit numerical values to simple empirical formulas. The flux is calculated for assorted values of $d_{r}$ using equation (1). Flux maxima for fixed $W$ are found numerically using equation (1) with $q=1$ over the range $0 \leq$ $W \leq 4$. These values are called $Q(1)$ and they are shown in Figure 1. Other calculations in the range $0<q<1$ have also been done [Whitehead and Salzig, 2001], and they readily produce values of flux between the two curves for $Q(0)$ and $Q(1)$.

[11] This gives us some comparison between the flux values in the range $0<q<1$. The greatest value of the ratio $Q(0) / Q(1)$ is 1.24 at $W=1.1$, and the average ratio for 40 equally spaced values in the range $0.1 \leq W \leq 4$ is 1.08 . Although this means that the flux equations (2) for zero potential vorticity are reasonably close fits for oceanographic applications, formulas with even closer fit to the flux for $q=1$ would be more appropriate because the layer of stagnant fluid in the upstream basin is the same depth as the Bernoulli Depth, which seems likely since the ocean water is stratified. If the bottom of the layer is the same depth as the bottom of the passage, water leaving the basin flows in a current along the left-hand wall toward the 


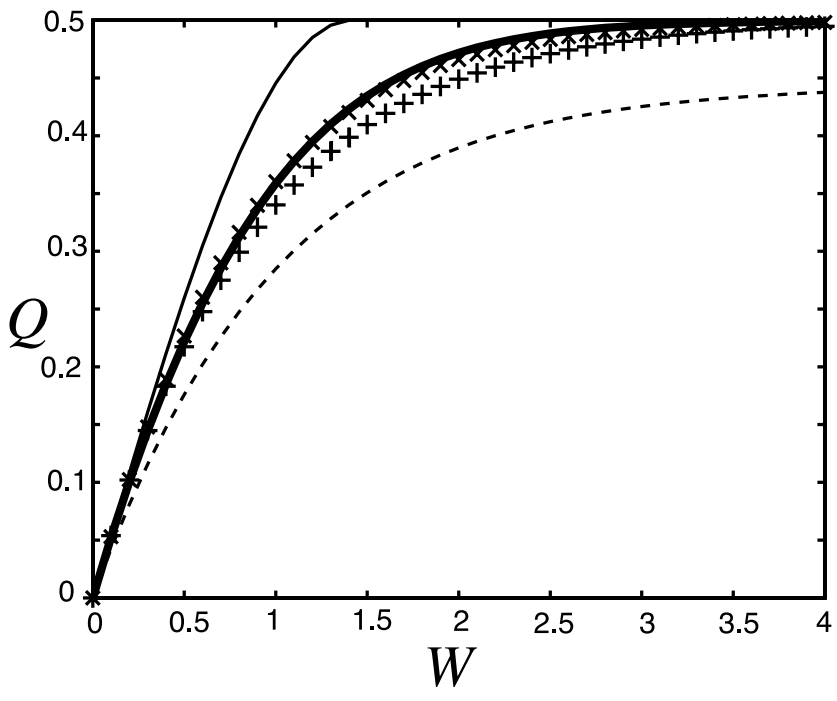

Figure 1. Flux curves from equations (1)-(4). The top solid curve is from equation (2) for zero potential vorticity. The bottom solid curve is for $\mathrm{Q}(1)$ (upstream layer depth equal to depth at the sill). The plus sign trajectory is from equation (3), and the $X$ values are from equation (4). The dashed line is from equation ( 1 ) for $q=1.5$ with maximum flux.

passage and out. This seems to be reasonable, since no specific mechanism is known to produce concentrated currents along the right-hand upstream wall.

[12] If such right-hand currents are present however, we could expect that $q>1$. In order to consider the entire range of possible potential vorticity for oceanic flows, the flux has been determined for critical flow for a value of $q=1.5$. There is a reduction of flux up to $20 \%$ compared to flux for $q=1$ as shown in Figure 1. However, $q=1.5$ possesses extremely unlikely upstream layer depths and flow patterns for the ocean. For example, the upstream potential vorticity depth satisfies $D_{\infty}^{*}=2 / 3 D^{*}$ so that the upstream layer is actually shallower than the layer flowing out through the passage. It is difficult to see how any continuously stratified fluid would allow this. Therefore, except for noting that such an extreme condition as $q=1.5$ reduces flux by up to $20 \%$, the focus of the rest of this study will be on flux with $q=1$.

[13] After considering a variety of simple formulas, we found that a good formula for a first approximation to $Q(1)$ is

$$
Q_{1}=0.5-0.5 e^{-1.0405 W} \text {. }
$$

This matches the two values $Q(1)=0,0.5$ at $W=0, \infty$ and minimizes the largest value of the scaled difference $\left|\frac{Q(1)-Q_{1}}{Q(1)}\right|$ to the value 0.0567 at $W=1.4$. Therefore this formula is less than $6 \%$ away from $Q(1)$ for all $W$.

[14] A more accurate formula has an additional term that allows a match with $d Q / d W$ at $W=0$. It is

$$
Q_{2}=0.5-0.6331 e^{-1.45 W}+0.1331 e^{-2.9 W} .
$$

[15] The exponential coefficients -1.45 and -2.9 minimize the largest value of the normalized difference $\left|\frac{Q(1)-Q_{2}}{Q(1)}\right|$ to a value of 0.0133 . This greatest difference is found at both $W=0.5$ and $W=2.0$. Therefore this formula is less than $1.4 \%$ away from $Q(1)$ for all $W$. Equation (1) for critical flux with $q=1$ and equations (2)-(4) are shown in Figure 1. It is obvious that equation (4) agrees closely with equation (1) with $q=1$. We assert that this formula, or equation (3) if a simpler one is needed, is best for ocean applications. In all events, equation (4) matches any conceivable upstream potential vorticity $(0<q<1.5)$ to better than $\pm 25 \%$, which is almost certainly the range for the ocean.

\section{Results}

[16] To illustrate the practical use of equation (4) in comparison to equation (2), they are both applied to nine ocean passages in Table 1 and Figure 2. This exercise is similar to that of Whitehead [1989, 1995], and in Table 3 and Figure 12 of Whitehead [1998], where hydrographic and bathymetric data were used to estimate, using our notation, the passage width $w^{*}$, depth of a fluid layer in an upstream basin $D^{*}$, a bulk density difference of the flowing layer $\Delta \rho^{*} / \rho^{*}$, and Coriolis parameter from rotation $f^{*}$. All these ocean passages have direct current meter measurements as a basis for their estimate of volume flux, so that comparison between an estimate using equations (2) and (4) and ocean current meter results can be made. The parameters are listed in Table 1.

[17] The Anagada Passage is added here since current meter results are now available. Also, hydrographic data from Hogg et al. [1999] give a clearer estimate of $D^{*}$ upstream of the Vema Channel. The volume flux estimate is considerably lower than the previous one (Vema Channel

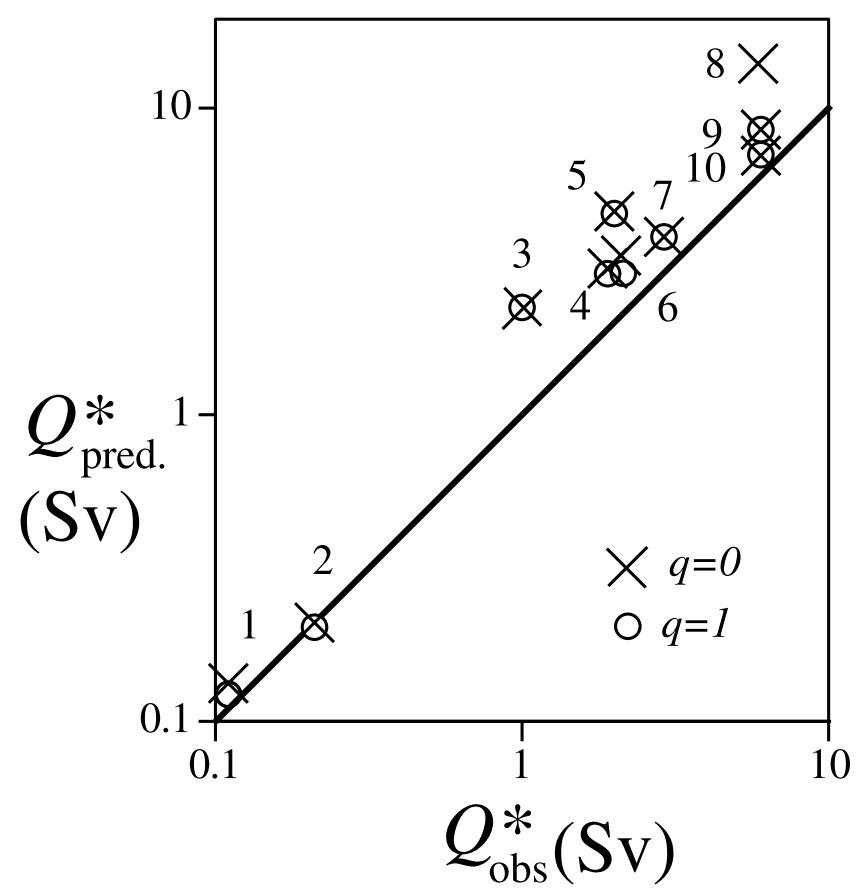

Figure 2. Comparison of zero and constant potential vorticity formulas (equations (2) and (4), respectively) with ocean measurements for nine passages with current meter measurements as listed in Table 1. 


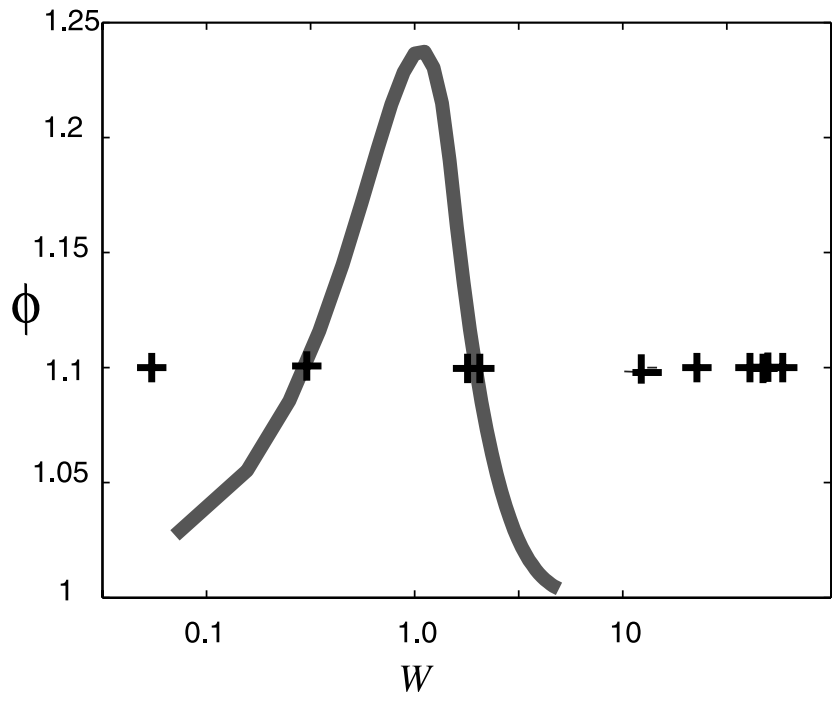

Figure 3. Relative flux difference $\phi=\left(Q(0)-Q_{2}\right) / Q_{2}$ as a function of passage width $W$. Widths for the ocean passages listed in Table 1 and in Figure 2 are shown by pluses.

with GEOSECS data), which used GEOSECS to estimate $D^{*}$. That case was known to be poorly approximated.

[18] Figure 2 shows that the estimates for flux in the ocean are very insensitive to whether $q$ is 0 or 1 . Why are the ocean cases so insensitive? The reason is that the widths $W$ of the listed passages do not fall within the range where the ratio is significantly larger than 1 . To illustrate this, Figure 3 shows the scaled difference between the zero and the constant potential vorticity formulas $\phi=\left(Q(0)-Q_{2}\right) / Q_{2}$ as a function of passage width $W$. This figure shows that $\phi>$ 1.1 only in the range $0.36<W<1.85$. There are only three ocean cases within this range, and they fall near the values where $\phi=1.1$. Therefore, in many cases, equation (2) is adequate, but at best, equations (3) and (4) are as good as needed to estimate inviscid steady flow over a passage with a flat bottom. One or the other might be useful for expressing flux through passages in ocean numerical models.

[19] Naturally, there are many additional factors in the ocean that can affect flux. Probably, the two most important factors are shape of the passage and friction. In addition, the effects of adjacent currents, mixing effects, and timedependent effects from waves or eddies would have quantifiable effects. For flows through ocean passages, it is not yet known which one (or ones) of these additional factors is most prominent in modifying flux rates from the first approximations given here.

[20] Acknowledgments. I thank Anna Nikolopoulos for substantial suggestions and an anonymous referee for suggesting Figure 3. Support is provided by the Ocean Sciences Section of the National Science Foundation under grant OCE-0325102. Woods Hole Oceanographic Institution contribution 11250

\section{References}

Dickson, R. R., E. M. Gmitrowicz, and A. J. Watson (1990), Deep water renewal in the northern Atlantic, Nature, 344, 848-850.

Gill, A. E. (1977), The hydraulics of rotating channel flow, J. Fluid Mech., $80,641-671$

Hall, M., M. S. McCartney, and J. A. Whitehead (1997), Antarctic bottom water flux in the equatorial western Atlantic, J. Phys. Oceanogr., 27, $1903-1926$.

Hogg, N. G., G. Siedler, and W. Zenk (1999), Circulation and variability at the southern boundary of the Brazil Basin, J. Phys. Oceanogr., 29, $145-$ 157.

MacCready, P., W. E. Johns, C. G. Rooth, D. M. Fratantoni, and R. A. Watlington (1999), Overflow into the deep Caribbean: Effects of plume variability, J. Geophys. Res., 104, 25,913-25,935.

McCartney, M. S., S. L. Bennett, and M. E. Woodgate-Jones (1991), Eastward flow through the Mid-Atlantic Ridge at $11^{\circ} \mathrm{N}$ and its influence on the abyss of the Eastern Basin, J. Phys. Oceanogr., 21, 1089-1120.

Mercier, H., and K. G. Speer (1998), Transport of bottom water in the Romanche Fracture Zone and the Chain Fracture Zone, J. Phys. Oceanogr., 28, 779-790.

Pratt, L. J., and L. Armi (1987), Hydraulic control of flows with nonuniform potential vorticity, J. Phys. Oceanogr., 17, 2016-2029.

Rudnick, D. L. (1997), Direct velocity measurements in the Samoan Passage, J. Geophys. Res., 102, 3293-3302.

Saunders, P. M. (1987), Flow through Discovery Gap, J. Phys. Oceanogr. 17, 631-643.

Saunders, P. M. (1990), Cold outflow from the Faeroe Bank Channel, J. Phys. Oceanogr., 20, 29-43.

Whitehead, J. A. (1989), Internal hydraulic control in rotating fluidsApplications to oceans, Geophys. Astrophys. Fluid Dyn., 48, 169-192.

Whitehead, J. A. (1995), Critical control by topography: Deep passages, straits and shelf fronts, in Topographic Effects in the Ocean, edited by P. Muller and D. Henderson, School of Ocean and Earth Sci. and Technol., Univ. of Hawai'i at Manoa, Honolulu.

Whitehead, J. A. (1998), Topographic control of ocean flows in deep passages and straits, Rev. Geophys., 36, 423-440.

Whitehead, J. A. (2003), Constant potential vorticity hydraulically controlled flow-complexities from passage shape, J. Phys. Oceanogr., 33, $305-312$.

Whitehead, J. A., and J. Salzig (2001), Rotating channel flow: Control and upstream currents, Geophys. Astrophys. Fluid Dyn., 95, 185-226.

Whitehead, J. A., A. Leetmaa, and R. A. Knox (1974), Rotating hydraulics of strait and sill flows, Geophys. Fluid Dyn., 6, 101-125.

J. A. Whitehead, Department of Physical Oceanography, Woods Hole Oceanographic Institution, MS 21, Woods Hole, MA 02543, USA. (jwhitehead@whoi.edu) 